\title{
Diffusion of High Energy Gamma-Rays through Matter.* II. Solution of the Diffusion Equation
}

\author{
L. L. Foldy AND R. K. OsBorn \\ Case Institute of Technology, Cleveland, Ohio
}

(Received September 11, 1950)

\begin{abstract}
An approximate solution of the diffusion equation derived by Foldy has been obtained for the case in which the total gamma-ray cross section is independent of energy. This is the case for essentially all materials in the energy region in which the derived diffusion equation is valid. For monoenergetic gamma-rays incident normally on a slab of material, the distribution in angle of gamma-rays of a given energy after traversing a large thickness of material is shown to be Gaussian with a breadth depending on the energy in accordance with a theorem derived by Foldy. Some numerical calculations for the case of $17-\mathrm{Mev}$ gammarays incident on water are presented to illustrate the nature of the results.
\end{abstract}

\section{INTRODUCTION}

$I^{\mathrm{N}}$ $\mathrm{N}$ the preceding paper ${ }^{1}$ (hereinafter referred to as $\mathrm{I}$ ) an equation was derived describing the diffusion of high energy gamma-rays through matter. This equation is applicable in the energy region extending from a few Mev to the critical energy at which radiation by the secondary electrons arising from the photoelectric effect and (more importantly) from pair production by the gamma-rays became important. In light materials the region of validity is a fairly wide energy range.

In the present paper a solution of this equation will be derived applicable to the energy region in which the total gamma-ray cross section is approximately independent of energy. Since there is always a minimum in the gamma-ray cross section as a function of energy, there is in all materials an energy region in which the total cross section will be substantially constant. The breadth of this region varies greatly from material to material, but an examination of the total cross-section curves given in I shows that actually, for each material over the energy range for which the derived transport equation is valid, the cross section may legitimately be regarded as approximately independent of energy. Hence the assumption of a constant total cross section represents really no further approximation to the diffusion equation.

Unfortunately, even with this simplification, the solution of the transport equation presents a formidable problem. Indeed, it will be necessary to make a further approximation with considerably less justification than any made previously. The exact nature of the approximation is described below; it consists in replacing a varying factor under the integral in the transport equation by a constant value. The factor actually varies from the value 2 at small angles to a value 1 at large angles. In replacing it by the constant value 2 , the results will be correspondingly inaccurate but only for gamma-rays much softer than the incident gamma-rays; for gamma-rays only slightly softer than the incident

* This work was supported in part by the AEC and by a grant-in-aid to one of the authors (L.L.F.) from the Scientific Research Society of America.

${ }^{1}$ L. L. Foldy, Phys. Rev. 80, 395 (1950), preceding paper. gamma-rays, there is no significant impairment of the results.

\section{SOLUTION OF THE TRANSPORT EQUATION}

The transport equation derived in $\mathbf{I}$ can be written as $\partial f(\sigma, \xi, \eta, \zeta) / \partial \zeta+\phi_{T} f(\sigma, \xi, \eta, \zeta)$

$$
\begin{aligned}
=\frac{1}{2 \pi} \int_{-\infty}^{\infty} \int_{-\infty}^{\infty} f\left(\sigma^{\prime}, \xi^{\prime}, \eta^{\prime}, \zeta\right)\left(\sigma^{\prime} / \sigma\right)^{2} \\
\quad \times\left[\sigma / \sigma^{\prime}+\sigma^{\prime} / \sigma\right] d \xi^{\prime} d \eta^{\prime},
\end{aligned}
$$

with

$$
\sigma^{\prime}=\sigma-\frac{1}{2}\left\{\left(\xi-\xi^{\prime}\right)^{2}+\left(\eta-\eta^{\prime}\right)^{2}\right\}
$$

where the total cross section, $\phi_{T}$, may, in accordance with the discussion in the introduction above, be regarded as constant. (For definitions of the symbols, reference should be made to $I$ ). We first transform the dependent variable by the transformation $\left(\sigma_{0}\right.$ is a constant to be defined later)

$$
g(\sigma, \xi, \eta, \zeta)=\left(\sigma / \sigma_{0}\right) f(\sigma, \xi, \eta, \zeta),
$$

which reduces Eq. (1) to the simpler form:

$$
\begin{aligned}
& \partial g(\sigma, \xi, \eta, \zeta) / \partial \zeta+\phi_{T} g(\sigma, \xi, \eta, \zeta) \\
& \quad=\frac{1}{2 \pi} \int_{-\infty}^{\infty} \int_{-\infty}^{\infty} g\left(\sigma^{\prime}, \xi^{\prime}, \eta^{\prime}, \zeta\right)\left[1+\left(\sigma^{\prime} / \sigma\right)^{2}\right] d \xi^{\prime} d \eta^{\prime}
\end{aligned}
$$

The approximation which we now make consists in replacing the term $\left[1+\left(\sigma^{\prime} / \sigma\right)^{2}\right]$ under the integral sign by the constant value 2 which reduces the equation to the form:

$$
\begin{aligned}
\partial g(\sigma, \xi, \eta, \zeta) / \partial \zeta & +\phi_{T} g(\sigma, \xi, \eta, \zeta) \\
& =\frac{1}{\pi} \int_{-\infty}^{\infty} \int_{-\infty}^{\infty} g\left(\sigma^{\prime}, \xi^{\prime}, \eta^{\prime}, \zeta\right) d \xi^{\prime} d \eta^{\prime}
\end{aligned}
$$

The method of solution which we shall employ makes use of Fourier transformations on the variables $\xi$ and $\eta$ and a Laplace transformation on the variable $\sigma$ :

$$
\begin{aligned}
g(\sigma, \xi, \eta, \zeta)=\int_{-\infty}^{\infty} \int_{-\infty}^{\infty} & \int_{-i \infty}^{i \infty} \gamma(s, \alpha, \beta, \zeta) \\
& \times \exp (i \alpha \xi+i \beta \eta+s \sigma) d s d \alpha d \beta
\end{aligned}
$$


Inserting this expression into Eq. (4), one finds that the transform function $\gamma$ must satisfy the equation

$$
\partial \gamma / \partial \zeta+\left\{\phi_{T}-(2 / s) \exp \left[-\left(\alpha^{2}+\beta^{2}\right) / 2 s\right]\right\} \gamma=0,
$$

with solution

$$
\begin{aligned}
& \gamma=G(\alpha, \beta, s) \exp \left\{-\phi_{T} \zeta+\right.(2 \zeta / s) \\
&\left.\times \exp \left[-\left(\alpha^{2}+\beta^{2}\right) / 2 s\right]\right\},
\end{aligned}
$$

where $G(\alpha, \beta, s)$ is an arbitrary function of its arguments.

The form of $G$ is fixed by the initial conditions, that is, the distribution function of the gamma-rays incident on the face of the slab of material, $\zeta=0$. We assume that the incident gamma-rays fall normally on the slab and are monoenergetic with softness $\sigma_{0}$. These conditions can be stated mathematically by writing

$$
g(\sigma, \xi, \eta, 0)=\delta\left(\sigma-\sigma_{0}\right) \delta(\xi) \delta(\eta),
$$

and this condition, in turn, will be satisfied provided we take $G$ to have the form

$$
G(\alpha, \beta, s)=e^{-s \sigma_{0}} /(2 \pi)^{3} i .
$$

The remainder of the problem then consists in substituting (8) and (10) into (6) and carrying out the integrations. We have

$$
\begin{aligned}
g(\sigma, \xi, \eta, \zeta)= & \frac{1}{(2 \pi)^{3} i} \int_{-\infty}^{\infty} \int_{-\infty}^{\infty} \int_{-i \infty}^{i \infty} \\
& \times \exp \left\{-\phi_{T} \zeta+s\left(\sigma-\sigma_{0}\right)+i \alpha \xi+i \beta \eta\right. \\
& \left.\quad+\frac{2 \zeta}{s} \exp \left[-\left(\alpha^{2}+\beta^{2}\right) / 2 s\right]\right\} d s d \alpha d \beta \\
= & \exp \left(-\phi_{T} \zeta\right) \cdot \sum_{m=0}^{\infty} g_{m} . \\
g_{m}= & \frac{1}{(2 \pi)^{3} i m !} \int_{-\infty}^{\infty} \int_{-\infty}^{\infty} \int_{-i \infty}^{i \infty}\left(\frac{2 \zeta}{s}\right)^{m} \\
& \quad \times \exp \left\{s\left(\sigma-\sigma_{0}\right)+i \alpha \xi+i \beta \eta\right. \\
& \left.\quad-m\left(\alpha^{2}+\beta^{2}\right) / 2 s\right\} d s d \alpha d \beta .
\end{aligned}
$$

The evaluation of the $g_{m}$ can be accomplished without difficulty. One has immediately

and

$$
g_{0}=\delta\left(\sigma-\sigma_{0}\right) \delta(\xi) \delta(\eta),
$$

$$
\begin{array}{r}
g_{1}=\frac{1}{(2 \pi)^{3} i} \int_{-\infty}^{\infty} \int_{\infty}^{\infty} \int_{-i \infty}^{i \infty} \frac{2 \zeta}{s} \exp \left\{s\left(\sigma-\sigma_{0}\right)+i \alpha \xi\right. \\
\left.+i \beta \eta-\left(\alpha^{2}+\beta^{2}\right) / 2 s\right\} d s d \alpha d \beta \\
=\frac{\zeta}{2 \pi^{2} i} \int_{-i \infty}^{i \infty} \exp \left\{s\left(\sigma-\sigma_{0}-\frac{\xi^{2}+\eta^{2}}{2}\right)\right\} d s \\
=-\delta\left(\sigma-\sigma_{0}-\frac{\xi^{2}+\eta^{2}}{2}\right) .
\end{array}
$$

For $m \geq 2$, the integrations of $\alpha$ and $\beta$ are easily carried out. The further integration on $s$ can be carried out by the use of Jordan's lemma and the method of residues; one obtains

$$
\begin{aligned}
g_{m}=\frac{1}{4 \pi^{2} i m \cdot m !} \int_{-i \infty}^{i \infty} s\left(\frac{2 \zeta}{s}\right)^{m} \\
\quad \times \exp \left[s\left(\sigma-\sigma_{0}-\frac{\xi^{2}+\eta^{2}}{2 m}\right)\right] d s \\
=\frac{(m-1)(2 \zeta)^{m}}{2 \pi(m !)^{2}}\left(\sigma-\sigma_{0}-\frac{\xi^{2}+\eta^{2}}{2 m}\right)_{*}^{m-2},
\end{aligned}
$$

where the asterisk subscript on the parenthesis indicates that the quantity inside the parenthesis is to be replaced by zero if it is negative.

The solution which we have obtained is, apart from the over-all exponential absorption factor, in the form of a power series in the thickness of material traversed. Each of the terms in the series has a rather simple physical interpretation. The first term represents the original gamma-rays which have filtered through to any depth without undergoing scattering or absorption. The second term, as can be seen from the correlation between energy and angle, corresponds to gamma-rays which have undergone only a single Compton scattering. The succeeding terms represent gamma-rays which have undergone 2, 3, etc. Compton scatterings in reaching the indicated depth.

An examination of the quantities $g_{m}$ for large $m$ reveals that they are closely related to the terms in the power series expansion of the zeroth-order Bessel function of imaginary argument. We write $g_{m}$ as

$$
g_{m}=-\frac{\partial^{2}}{\partial \sigma \partial\left(\vartheta^{2}\right)}\left\{\frac{\left[2 \zeta\left(\sigma-\sigma_{0}\right)\right]^{m}}{\pi(m !)^{2}}\left[1-\frac{\vartheta^{2}}{2 m\left(\sigma-\sigma_{0}\right)}\right]_{*}^{m}\right\},
$$

and note that for large $m$ it may be approximated by

$$
g_{m} \sim-\frac{\partial^{2}}{\partial \sigma \partial\left(\vartheta^{2}\right)}\left\{\frac{(\rho / 2)^{2 m}}{\pi(m !)^{2}} \exp \left[-\vartheta^{2} / 2\left(\sigma-\sigma_{0}\right)\right]\right\},
$$

where

$$
\rho=\left[8 \zeta\left(\sigma-\sigma_{0}\right)\right]^{\natural} .
$$

Now the zeroth-order Bessel function of imaginary argument, $I_{0}(\rho)$ has the power series expansion

$$
I_{0}(\rho)=\sum_{m=0}^{\infty}(\rho / 2)^{m} /(m !)^{2} .
$$

We can exploit this close relationship between the two 
series by writing

$$
\begin{aligned}
\sum_{m=2}^{\infty} g_{m}= & -\frac{1}{\pi} \frac{\partial^{2}}{\partial \sigma \partial\left(\vartheta^{2}\right)}\left\{\left[I_{0}(\rho)-1-\rho^{2} / 4\right]\right. \\
& \left.\times \exp \left[-\vartheta^{2} / 2\left(\sigma-\sigma_{0}\right)\right]\right\} \\
& +\sum_{m=2}^{\infty} \frac{(\rho / 2)^{m}}{(m !)^{2}}\left\{\left[1-\vartheta^{2} / 2 m\left(\sigma-\sigma_{0}\right)\right]_{*}{ }^{m}\right. \\
= & \left.-\exp \left[-\vartheta^{2} / 2\left(\sigma-\sigma_{0}\right)\right]\right\} \\
& \frac{\partial}{\partial \sigma}\left\{\zeta\left[\frac{I_{0}(\rho)-1}{\rho^{2} / 4}-1\right]\right. \\
& \left.\times \exp \left[-\vartheta^{2} / 2\left(\sigma-\sigma_{0}\right)\right]\right\} \\
& -\frac{1}{\pi} \frac{\partial^{2}}{\partial \sigma \partial\left(\vartheta^{2}\right)} \sum_{m=2}^{\infty} \frac{(\rho / 2)^{m}}{(m !)^{2}} \\
& \times\left\{\left[1-\vartheta^{2} / 2 m\left(\sigma-\sigma_{0}\right)\right]_{*}{ }^{m}\right. \\
& \left.-\exp \left[-\vartheta^{2} / 2\left(\sigma-\sigma_{0}\right)\right]\right\} .
\end{aligned}
$$

Inserting these results into (11) and (3) we obtain two equivalent forms for the distribution function:

$$
\begin{aligned}
& f(\sigma, \xi, \eta, \zeta)= {\left[\delta\left(\sigma-\sigma_{0}\right) \delta(\xi) \delta(\eta)+\frac{\zeta \sigma_{0}}{\pi \sigma} \delta\left(\sigma-\sigma_{0}-\frac{1}{2} \vartheta^{2}\right)\right.} \\
&+\frac{\sigma_{0}}{\sigma} \sum_{m=2}^{\infty} \frac{(m-1)(2 \zeta)^{m}}{(m !)^{2}} \\
&\left.\times\left(\sigma-\sigma_{0}-\vartheta^{2} / 2 m\right)_{*}{ }^{m-2}\right] \exp \left(-\phi_{T} \zeta\right) \\
&= {\left[\delta\left(\sigma-\sigma_{0}\right) \delta(\xi) \delta(\eta)+\frac{\zeta \sigma_{0}}{\pi \sigma} \delta\left(\sigma-\sigma_{0}-\frac{1}{2} \vartheta^{2}\right)\right.} \\
&+\frac{\zeta \sigma_{0}}{\pi \sigma} \frac{\partial}{\partial \sigma}\left\{\left[\frac{I_{0}(\rho)-1}{\rho^{2} / 4}-1\right]\right. \\
&\left.\left.\times \exp \left[-\vartheta^{2} / 2\left(\sigma-\sigma_{0}\right)\right]\right\}\right] \exp \left(-\phi_{T} \zeta\right) \\
&+\left[\frac { \sigma _ { 0 } } { \pi \sigma } \frac { \partial ^ { 2 } } { \partial \sigma \partial ( \vartheta ^ { 2 } ) } \left\{\sum_{m=2}^{\infty} \frac{(\rho / 2)^{m}}{(m !)^{2}}\right.\right. \\
& \times\left\{\exp \left[-\vartheta^{2} / 2\left(\sigma-\sigma_{0}\right)\right]\right. \\
&\left.\left.\left.-\left[1-\vartheta^{2} / 2 m\left(\sigma-\sigma_{0}\right)\right]_{*}{ }^{m}\right\}\right\}\right] \\
& \times \exp \left(-\phi_{T} \zeta\right) .
\end{aligned}
$$

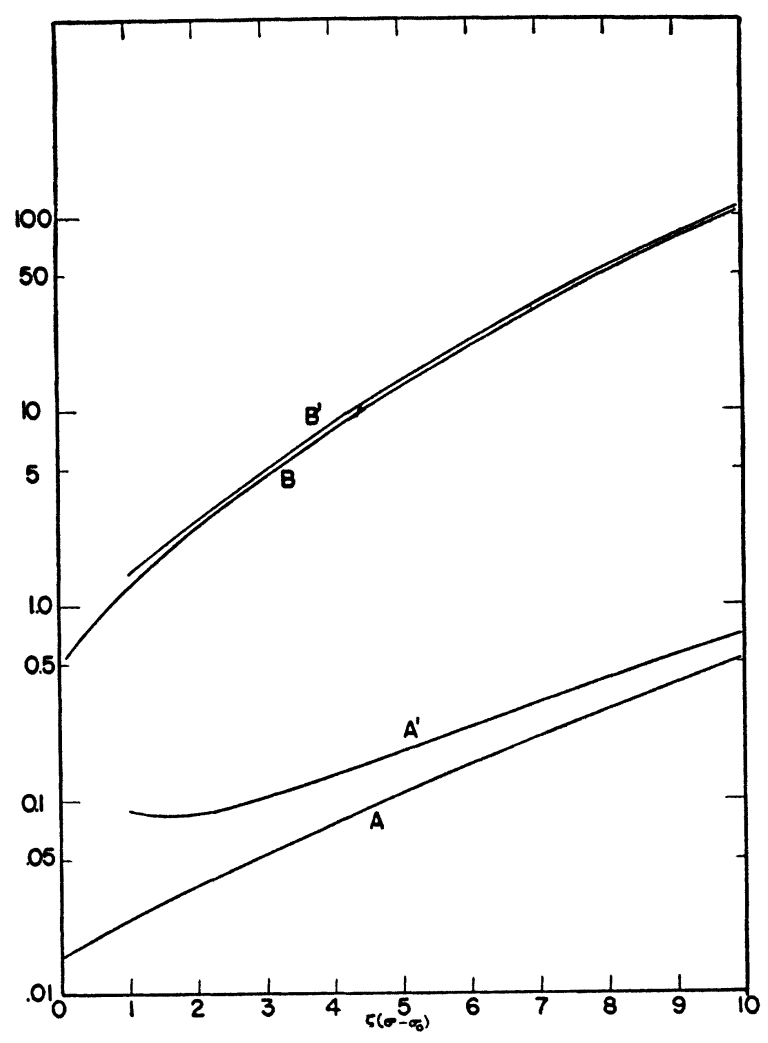

FIg. 1. Functions representing energy spectrum of multiply scattered gamma-rays apart from factor $\sigma_{0} / \sigma .(A)$ Spectrum on axis, $\left(A^{\prime}\right)$ asymptotic approximation for spectrum on axis, $(B)$ spectrum integrated over all angles, $\left(B^{\prime}\right)$ asymptotic approximation for spectrum integrated over all angles.

The second form has the advantage that in two important cases the last term (the summation) can be neglected compared to the other terms. First, if $\vartheta^{2} / 2\left(\sigma-\sigma_{0}\right) \ll 1$ then the expression in braces in the summation is very small for every term and the summation can be neglected. Secondly, for large values of $\zeta\left(\sigma-\sigma_{0}\right)$, one finds that most of the contributions to the series $\sum_{m=2}^{\infty} g_{m}$ comes from terms where $m \sim\left[2 \zeta\left(\sigma-\sigma_{0}\right)\right]^{1}$; hence if $\vartheta^{2} / 2\left(\sigma-\sigma_{0}\right) \ll\left[2 \zeta\left(\sigma-\sigma_{0}\right)\right]^{2}$, the use of the approximate expression (16) for $g_{m}$ is justified and the summation can again be neglected. Hence in these cases

$$
\begin{array}{r}
f(\sigma, \xi, \eta, \zeta)=\left[\delta\left(\sigma-\sigma_{0}\right) \delta(\xi) \delta(\eta)+\frac{\zeta \sigma_{0}}{\pi \sigma} \delta\left(\sigma-\sigma_{0}-\frac{1}{2} \vartheta^{2}\right)\right. \\
+\frac{\zeta \sigma_{0}}{\pi \sigma} \frac{\partial}{\partial \sigma}\left\{\left[\frac{I_{0}(\rho)-1}{\rho^{2} / 4}-1\right]\right. \\
\left.\left.\quad \times \exp \left[-\vartheta^{2} / 2\left(\sigma-\sigma_{0}\right)\right]\right\}\right] \\
\times \exp \left(-\phi_{T} \zeta\right) .
\end{array}
$$


On the axis, $\vartheta=0$, the distribution function takes the simpler form

$$
\begin{aligned}
& f(\sigma, 0,0, \zeta)=\left[\delta\left(\sigma-\sigma_{0}\right) \delta(0) \delta(0)+\frac{\zeta \sigma_{0}}{\pi \sigma} \delta\left(\sigma-\sigma_{0}\right)\right. \\
& \left.+\frac{32 \zeta^{2} \sigma_{0}}{\pi \sigma}\left\{\frac{1-I_{0}(\rho)+\frac{1}{2} \rho I_{1}(\rho)}{\rho^{4}}\right\}\right] \exp \left(-\phi_{T} \zeta\right) .
\end{aligned}
$$

To show the distribution in energy of the multiply scattered gamma-rays on the axis, we have plotted in Fig. 1 as curve $A$ the factor in braces in (21) as a function of $\zeta\left(\sigma-\sigma_{0}\right)$. Aside from the additional factor $\sigma_{0} / \sigma$ this shows the relative distribution in energy.

The distribution of the gamma-rays per unit energy interval (rather than unit softness interval) can be obtained from the above formulas by simply multiplying by $\sigma^{2}$.

\section{ASYMPTOTIC DISTRIBUTION}

If $\rho=\left[8 \zeta\left(\sigma-\sigma_{0}\right)\right]^{t}$ is not only large compared to $\vartheta^{2} / 2\left(\sigma-\sigma_{0}\right)$ but is also large compared to unity, then it is permissible to replace the Bessel function occurring in (20) by its asymptotic form. Furthermore, after the differentiation with respect to $\sigma$ has been carried out, one can obtain the asymptotic form of the distribution function by neglecting all terms but the one which is dominant when $\left[8 \zeta\left(\sigma-\sigma_{0}\right)\right]^{t}$ is very large. The resultant asymptotic form is found to be

$$
\begin{aligned}
f(\sigma, \xi, \eta, \zeta) \sim \frac{32 \zeta^{2} \sigma_{0}}{\pi \sigma} \exp [ & \left.-\vartheta^{2} / 2\left(\sigma-\sigma_{0}\right)\right] \\
& \times\left\{\frac{\frac{1}{2} e^{\rho}}{(2 \pi)^{\frac{1}{2}} \rho^{7 / 2}}\right\} \exp \left(-\phi_{T} \zeta\right),
\end{aligned}
$$

with the validity condition

$$
\left.\rho=\left[8 \zeta\left(\sigma-\sigma_{0}\right)\right]\right]^{\natural} \gg 1+\vartheta^{2} / 2\left(\sigma-\sigma_{0}\right) .
$$

It will be noted that the distribution in angle of gammarays of a given softness is just the Gaussian distribution derived by elementary arguments in $\mathbf{I}$. The distribution in energy on the axis (except again for the factor $\sigma_{0} / \sigma$ ) according to this asymptotic formula is given by the curve $A^{\prime}$ in Fig. 1.

\section{INTEGRATION OVER ANGLES}

To obtain the flux of gamma-rays at any depth independent of the direction in which they are travelling one may simply integrate the distribution (19) over all angles. One obtains the result:

$$
\begin{aligned}
F(\sigma, \zeta)=\iint f d \xi d \eta= & \delta\left(\sigma-\sigma_{0}\right) \exp \left(-\phi_{T} \zeta\right) \\
& +\frac{4 \zeta \sigma_{0} \exp \left(-\phi_{T} \zeta\right)}{\sigma}\left\{\frac{I_{1}(\rho)}{\rho}\right\} .
\end{aligned}
$$

It is easily found that the integration over angles of the approximate formula (20) gives exactly the same result, so that the approximation involved in dropping the last summation in (19) corresponds only to a redistribution in angle of gamma-rays of given energy at any depth without a change in their total number.

The first term in (23) represents, of course, the gamma-rays which have reached the depth $\zeta$ unscattered and unabsorbed, while the second term represents the scattered gamma-rays. The distribution in energy of the latter as given by the factor in braces (neglecting again the factor $\sigma_{0} / \sigma$ ) is plotted as curve $B$ in Fig. 1 as a function of $\zeta\left(\sigma-\sigma_{0}\right)$. When $\zeta\left(\sigma-\sigma_{0}\right)$ is very large compared with unity, the Bessel function can be replaced by its asymptotic form giving the distribution function

$$
F(\sigma, \zeta) \sim \frac{4 \zeta \sigma_{0} \exp \left(-\phi_{T} \zeta\right)}{\sigma}\left\{\frac{e^{\rho}}{(2 \pi)^{\frac{1}{\rho} \rho^{\rho}}}\right\} .
$$

The factor here in braces is also plotted in Fig. 1 as curve $B^{\prime}$.

\section{CALCULATIONS OF ANGULAR DISTRIBUTION}

In order to see in greater detail the angular distribution of the multiply scattered gamma-rays, some
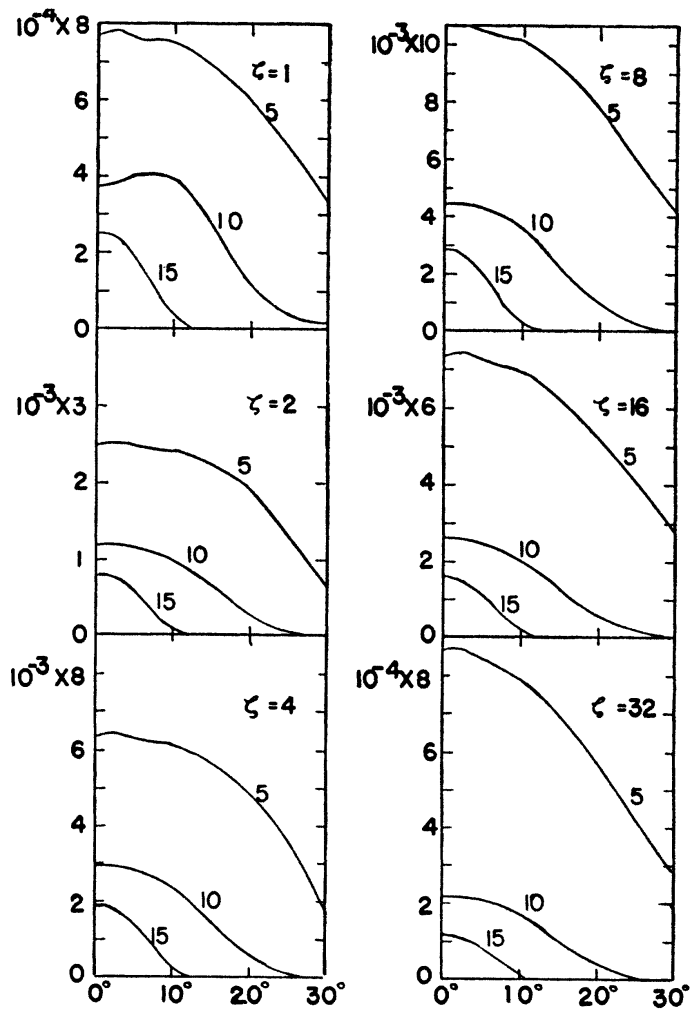

Fig. 2. Distribution in angle of multiply scattered gamma-rays of energies of 5,10 , and $15 \mathrm{Mev}$ at various depths in material for 17-Mev gamma-rays incident on water. Note variations in vertical scales which represent the number of gamma-rays per unit solid angle per Mev energy interval per incident gamma-ray. 
numerical calculations on the basis of Eq. (20) have been performed for incident gamma-rays of $17-\mathrm{Mev}$ energy diffusing through water. The curves of angular distribution as given by the number of gamma-rays per unit energy interval (energy measured in Mev) and per unit solid angle are plotted in Fig. 2 for energies of 15,10 , and $5 \mathrm{Mev}$ and $\zeta=1,2,4,8,16$, and 32 . It will be noted that the angular distribution is very close to Gaussian even for relatively small thicknesses of material.

For a complete picture of the energy and angular distribution one must add to the above curves the contributions of the two terms containing delta-functions in (20) representing the unscattered and singly scattered gamma-rays.

\section{CONCLUDING REMARKS}

The principal source of error in the solution obtained above results from the approximation made in going from Eq. (4) to Eq. (5). Going back to the fundamental equations, this corresponds to approximating the factor $\frac{1}{2}\left(\epsilon^{\prime} / \epsilon\right)^{2}\left[\epsilon / \epsilon^{\prime}+\epsilon^{\prime} / \epsilon\right]$ in the Klein-Nishina formula [I, Eq. (3)] by $\epsilon^{\prime} / \epsilon$ (neglecting the term in $\sin ^{2} \Theta$ ). The extent of this error is shown in Fig. 3 where both factors are plotted. Some improvement in the results might be obtained if one used in place of $\epsilon^{\prime} / \epsilon$, the quantity $c \epsilon^{\prime} / \epsilon$ where $c$ is a number lying between 0.5 and 1.

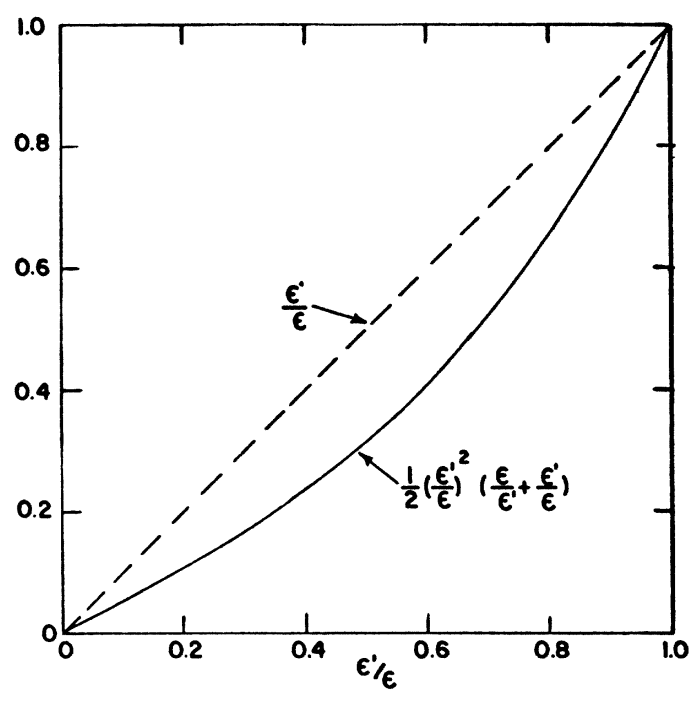

Fig. 3. Extent of approximation to Klein-Nishina formula involved in solution of the diffusion equation.

It would be of value to determine the corrections due to the difference between the approximate expression used and the correct expression by a perturbation treatment. A calculation of this type is planned for the future.

We wish to express our thanks to Mr. G. F. Bing for his aid with the numerical calculations.

\title{
Measurements of Gamma-Ray Absorption Coefficients*†
}

Charlotte Meaker Davisson $\ddagger$ and Robley D. Evans

Massachusetts Institute of Technology, Cambridge, Massachusetts

(Received December 5, 1949)

\begin{abstract}
The absorption of $\gamma$-rays in $\mathrm{Al}, \mathrm{Cu}, \mathrm{Sn}, \mathrm{Ta}$, and $\mathrm{Pb}$ was measured using $\gamma$-rays from $\mathrm{I}^{131}, \mathrm{Cu}^{64}, \mathrm{Mn}^{54}$, $\mathrm{Co}^{60}, \mathrm{Zn}^{65}$, and $\mathrm{Na}^{24}$. Tests showed that scattering from nearby objects and from the absorber were negligible. Tests of the absorption of radium $\gamma$-rays in $\mathrm{Pb}$ showed good agreement with other workers and with theory. The absorption curves of $I^{131}$ showed the presence of a $0.65-\mathrm{Mev} \gamma$-ray about 15 percent as abundant as the $0.367-\mathrm{Mev} \gamma$-ray. Absorption coefficients measured with the other sources showed agreement within 0.5 percent to 2 percent with theory. An anomalous absorption coefficient ( 5 percent less than expected) with tantalum absorber $(Z=73)$ and $\mathrm{Zn}^{65}$ and $\mathrm{Co}^{60}$ sources $(h \nu \sim 1.2 \mathrm{Mev})$ needs reinvestigation.
\end{abstract}

\section{INTRODUCTION}

W ITH the increasing use of radioactive materials, it is becoming important to know with greater accuracy how the interaction of $\gamma$-rays with matter varies with $\gamma$-ray energy and with atomic number. In the energy range of most radioactive $\gamma$-rays, that is, from $0.1 \mathrm{Mev}$ to $6 \mathrm{Mev}$, the processes to be considered are the Compton effect, the photoelectric effect, and

${ }^{*}$ Assisted by the joint program of the ONR and AEC.

+ Presented at the April, 1948, meetings of the American Physical Society.

$\$$ Present address: care of Dr. J. W. Davisson, Crystal Branch, Naval Research Laboratory, Washington 20, D. C. pair production. Theoretical analyses of these processes have been made, and from them values of absorption coefficients can be found for comparison with experiment. A summary of most of these theories has been given by Heitler. ${ }^{1}$ We have made a detailed study of the results of the theories and shall publish elsewhere ${ }^{2}$ our calculations in the form of equations, tables, and curves.

Early experimental studies of absorption coefficients

\footnotetext{
${ }^{1}$ W. Heitler, The Quantum Theory of Radiation (Oxford University Press, London, 1936), pp. 119 et seq.

2 C. M. Davisson and R. D. Evans, Revs. Modern Phys. (to be published).
} 